\title{
Search of new type of spatial organization of nucleic acids in human genome
}

\author{
Anastasia Zamoskovtseva \\ ICBFM SB RAS, Novosibirsk, \\ Russia, NSU, Novosibirsk, Russia \\ a.zamoskovtseva@g.nsu.ru
}

\author{
Marsel Kabilov \\ ICBFM SB RAS, Novosibirsk, \\ Russia,kabilov@niboch.ncs.ru \\ Alexander Lomzov \\ ICBFM SB RAS, Novosibirsk, \\ Russia \\ lomzov@niboch.ncs.ru
}

\author{
Dmitrii Pyshnyi \\ ICBFM SB RAS, Novosibirsk, \\ Russia,pyshnyi@niboch.ncs.ru
}

\begin{abstract}
It is well-known that nucleic acids perform regulatory functions in various molecular biological processes. Recently, a new type of spatial organization of nucleic acids formed by two duplex fragments and called a TW-type pseudoknot (twiplex) was found. In this work we have analyzed human genome to find these complexes. We developed an algorithm for bioinformatic search on the nucleotide sequences which potentially can form twiplex. The belonging of the obtained sequences to genes, introns or exons was analyzed.
\end{abstract}

Keywords - nucleic acids complexes, algorithm, human genome

Motivation and aim

\section{Motivation}

One of the functions of nucleic acids is regulation of various biological processes. In many ways, it is determined by the secondary and tertiary structure. Recently, a new type of spatial organization of nucleic acids was discovered in the ICBFM SB RAS. This complex is formed by two duplex fragments and called a TW-type pseudoknot (twiplex). It can be formed by single or two DNA or RNA chain, or in DNA / RNA intermolecular complex.

Aim

The aim of the work is to determine the possibility of implementation of twiplex structures in living systems.
We developed an algorithm for bioinformatic search on the nucleotide sequences which potentially can form twiplex. The method was applied for the human genome analysis. We consider structures with double-stranded blocks of the length from 5 to 20 base pairs. Additional selection of sequences was carried out in order to exclude sequences forming alternative secondary structures.

\section{Results}

Genome regions potentially capable for twiplexes formation have been detected. We analyzed the belonging of the obtained sequences to genes, introns or exons. Experimental studies have begun to study the physicochemical and molecular biological properties of selected nucleotide sequences.

\section{ACKNOWLEDGMENT}

Supported by the RFBR (20-04-00719) and by State project No. A-0309-2016-0004. 